Journal of

Neurolnterventional Surgery

In vitro experiments of cerebral blood flow during aspiration thrombectomy: Potential effects on cerebral perfusion pressure and collateral flow.

\begin{tabular}{|r|l|}
\hline Journal: & Journal of NeuroInterventional Surgery \\
\hline Manuscript ID: & neurintsurg-2015-011909.R2 \\
\hline Article Type: & Original research \\
\hline Keywords: & Stroke, Thrombectomy, Blood Flow, Catheter \\
\hline \multicolumn{2}{|l}{} \\
\hline
\end{tabular}

SCHOLARONE ${ }^{m}$

Manuscripts 


\section{In vitro experiments of cerebral blood flow during aspiration thrombectomy: Potential effects on cerebral perfusion pressure and collateral flow.}

Keywords: Stroke, recanalization, thrombectomy, aspiration, blood flow

Word count: 2140 


\begin{abstract}
Background: Mechanical thrombectomy with stenttriever devices is associated with significantly better outcomes than thrombolysis alone in the treatment of acute ischaemic stroke. Thrombus aspiration achieves high patency rates, but clinical outcomes are variable. The aim of this study was to examine the effect of different suction conditions on perfusate flow during aspiration thrombectomy.
\end{abstract}

Materials: A computational fluid dynamics model of an aspiration device within a patent and occluded blood vessel was used to simulate flow characteristics using fluid flow solver software (OpenFOAM, OpenCFD Ltd). A physical particulate-flow model of a patent vessel and a vessel occluded by thrombus was then used to visualize flow direction and measure flow rates with the aspiration catheter placed 1$10 \mathrm{~mm}$ proximal of the thrombus, and recorded on video.

Results: The mathematical model predicted that in a patent vessel perfusate is drawn from upstream of the catheter tip, while in an occluded system perfusate is drawn from the vessel proximal to the device tip, with no traction on the occlusion distal of the tip. The in-vitro experiments confirmed the predictions of this model. In the occluded vessel aspiration had no effect on the thrombus unless the tip of the catheter was in direct contact with the thrombus.

Conclusion: These experiments suggest that aspiration is only effective if the catheter tip is in direct contact with the thrombus. If the catheter tip is not in contact with the thrombus aspirate is drawn from the vessels proximal of the occlusion. This could affect collateral flow in vivo. 


\section{Introduction}

Early restoration of blood flow to the ischaemic brain is pivotal to good recovery and improved outcomes in ischaemic stroke patients ${ }^{1}$. Recanalization rates with intravenous thrombolysis are poor in patients with occlusion of a large intracerebral artery $(30 \%$ in basilar artery occlusions, $30 \%$ in proximal middle cerebral artery occlusions and $6 \%$ in terminal internal carotid artery occlusions) ${ }^{2}$. Endovascular treatment $(E V T)$ is associated with considerably higher recanalization rates than intravenous thrombolysis ${ }^{1}$. The most commonly used methods for EVT are mechanical thrombectomy using a stenttriever device and thrombus aspiration. Recently presented randomized controlled trials have shown significantly better outcomes for mechanical thrombectomy compared to control when stentrievers were used $^{3-7}$. Aspiration devices achieve consistently high recanalization rates (68-100\%), but clinical outcomes with aspiration are variable ${ }^{8}$ with very poor recovery rates in four studies ${ }^{9-12}$ and good outcomes in others ${ }^{13-17}$. Recanalization alone may not ensure good outcomes, if reperfusion is not achieved ${ }^{18}$.

In vitro experiments can be a useful tool for the study of specific attributes of device performance. Experiments in an in vitro model have previously shown that suction thrombectomy is considerably faster than thrombectomy using a stenttirever device but associated with a higher incidence of thrombus fragmentation ${ }^{19}$.

The aim of this study was to examine perfusate flow patterns during aspiration thrombectomy in an in-vitro system to investigate the effects of different suction conditions (flow rate, suction pressure, and distance from the thrombus) on flow in a model of occluded and unoccluded cerebral arteries. 


\section{Methods}

A mathematical model of blood flow in a blocked cerebral blood vessel was

developed. A physical flow model of suction thrombectomy in an occluded and unoccluded vessel was then set up to visualize flow patterns and to assess the effects of different aspiration conditions in vitro.

\section{The mathematical model}

A computational fluid dynamics (CFD) model was used to simulate the fluid flow characteristics of a typical catheter used for our experiments. A model of a cylinder within a cylinder was chosen to represent a plain catheter within an artery. The dimensions of the catheter cylinder were based on diameters of the catheters used (see below); approximately $1.4 \mathrm{~mm}$ OD and $0.89 \mathrm{~mm}$ ID. The artery cylinder was approximately $3 \mathrm{~mm}$ in diameter and the distal surface positioned $10 \mathrm{~mm}$ from the distal tip of the catheter cylinder. This model assumed laminar flow based on calculated Reynolds numbers that were in the order of thousands. Geometries of the fluid-flow system were created, meshed and run using the fluid-flow solver OpenFOAM (@ OpenCFD Ltd.).

\section{The physical flow model}

The model was set up as in Figure 1 incorporating a glass model of the MCA with an internal diameter of $2.4 \mathrm{~mm}$ and a downstream pinch point diameter of $2 \mathrm{~mm}$ where the thrombus was placed. A haemostatic valve proximal to the glass model was used to introduce catheters into the system. The system was adapted from a model described previously ${ }^{19}$ and primed at a mean arterial pressure of $90 \mathrm{~mm} \mathrm{Hg}$ by 
elevating the fluid reservoir $122 \mathrm{~cm}$ above the model. Two devices were used to create suction within the system; a syringe pump (Fusion 200 Touch syringe pump, KR Analyitical Ltd, UK) was used for generating low flow rates and a vacuum pump (Welch Vacuum Technology 2546B-01) for higher flow rates.

The system was primed with perfusate containing haematoxylin particles to allow visualization of flow patterns. This was prepared as follows: $1 \mathrm{ml}$ of haematoxylin solution (Gill no.3 - GHS316-Sigma Aldrich) was added to $800 \mathrm{ml}$ of water and left for 1 hour to produce clumps. The solution was stirred and allowed to settle for 30 minutes repeatedly to obtain a suspension containing smaller particles. This produced a particulate that behaved like a colloid over the time periods of the experiments without significant settling and allowed even small movements of flow to be observed.

\section{Thrombus production}

Fresh sheep's blood was obtained from a local abattoir in a closed container. The blood was allowed to clot naturally. After clotting the blood was cut into small pieces and snap-frozen in liquid nitrogen before being placed in a freezer at $-20^{\circ} \mathrm{C}$. Thrombi were taken from the freezer in small quantities and allowed to thaw gradually in physiological saline solution at $4^{\circ} \mathrm{C}$. On thawing the thrombus was cut down with a scalpel until it weighed between $12-14 \mathrm{~g}$ after drying on a paper towel. This weight had been arrived at experientially and provided a clot of a size that engaged well within the model system. Once primed, the clot was introduced into the system and pushed to the stricture point using brief flow from the pump. The model was then 
flushed with perfusate to remove solubilised blood from around the clot to allow accurate visualisation.

\section{Flow observation in a blocked system}

A catheter was introduced into the system via the haemostatic valve, with the tip at a distance of $1,2,2.5$, and $10 \mathrm{~mm}$ from the clot. Air was removed from the system and the particulate allowed to equilibrate. At an appropriate point the pump was switched on and the flow in the area of the catheter tip and clot was observed over a minimum of 30 seconds using a video camera (Samsung, 42X INTELLI-ZOOM, 24fps). Flow rates used ranged from 5 to $40 \mathrm{kPa}$ using the vacuum pump and 2,5 and $8 \mathrm{ml} / \mathrm{min}$ using the syringe pump. The flow rates were calculated by analysis of video data from the closed system. Fifteen particles were randomly selected and time taken for each particle to travel $2 \mathrm{~cm}$ was timed using a stopwatch.

\section{Catheters}

Two catheters were used, a general purpose Fr4 MC 0.035" (0.89mm) (Kimal, Uxbridge, UK) and a NeuroSlider*17 (0.42mm) microcatheter (Acandis, Pforzheim, Germany). The size of the catheter was chosen to match the size of the target vessel (2.4 $\mathrm{mm}$ ) using recommendations for catheter size for currently used catheters in vivo $^{20} 21$.

\section{Results}

\section{Mathematical model of suction thrombectomy}

Results of the modelling showed that in an unobstructed system (cerebral artery not occluded by a thrombus) flow is drawn from upstream of the tip of a device when 
suction is applied. In an occluded system (cerebral artery obstructed by a thrombus) the mathematical model showed that flow came from behind the tip of the device when suction was applied, and that there was no traction effect on flow distal of the tip. The scaled-up stream tracer plots of an occluded model in Figure 2a show torsional movement downstream of catheter tip but no visible torsional movement from seed points $3 \mathrm{~mm}$ upstream of the catheter tip. The only $x$ and $y$-direction flow pattern is seen as the fluid flows past the outside of the catheter in the negative zdirection, bending around the distal end of the catheter tip toward the inside of the device. The tracer plots in figure $2 \mathrm{~b}$ which comes from the unoccluded mathematical model shows clear flow from the same $3 \mathrm{~mm}$ seed points upstream of the catheter tip as well as flow from the negative $z$ direction.

\section{Physical model}

At all flow rates and separation distances, fluid was clearly drawn from behind the catheter as predicted by the mathematical model (Figure 2) and not from in front when a clot was present, which is illustrated in Figure 3a. As the flow became steady an area of turbulence was observed in front of the catheter tip and this area of turbulence increased with increasing flow rates (Figure 3b). The turbulence had no effect on clot removal at any distance from the catheter, the catheter needed to be touching the clot before suction had any effect. At further separation distances, no clot movement or engagement with the suction catheter was observed in any of the experiments.

When suction was applied to the devices in an occluded model, flow only occurred from behind the tip of the device, Figure 4a. When suction was applied to the devices in the absence of a thrombus flow from the front of the device was restored 
but there was still a clear but less dominant flow present from behind the catheter tip, Figure $4 b$.

\title{
Discussion
}

Mathematical modelling of blood flow in an occluded vessel during suction through a catheter showed an area of turbulence between the clot and the catheter tip with blood drawn from the vessel segment proximal to the catheter tip and no effect of the suction on the clot or on the vessel segment distal to the clot. These findings from our mathematical model are confirmed by in-vitro experiments performed in a flow model.

The key finding of this in-vitro research is that catheter-based suction only engaged the thrombus if the tip of the catheter was in direct contact with the thrombus. When the thrombus was not engaged in the tip of the suction catheter, suction removed only perfusate, and produced no traction forces on the thrombus. Perfusate removed by suction came from behind, e.g. the vessel section proximal to the catheter tip. This has potentially significant implications for clinical practice.

\begin{abstract}
After occlusion of a large artery, the ensuing drop in perfusion pressure distally generates a pressure gradient between neighbouring arterial fields, resulting in changes in flow direction and rate; collateral flow changes occur almost immediately, within 1-4 seconds ${ }^{22}$. In our experimental model we observed that non-engagement of the suction catheter with the thrombus results in removal of the perfusate proximal
\end{abstract}


of the catheter tip. In a clinical scenario this could lead to flow reversal in the proximal/collateral vessels, leading to a decrease in the perfusion pressure within the ischemic penumbra. This in turn would produce a vicious cycle of further reduction of blood flow in other collateral vessels, thereby incorporating a significant proportion of the salvageable penumbra into the ischemic core. Such flow reductions could be particularly relevant during aspiration in small calibre M2 segment of the middle cerebral artery (MCA) or in the basilar artery. Placement of the aspiration catheter within the distal M1/proximal M2 could cause flow reversal within the other viable MCA branches including the lateral and medial lenticulostriate perforator vessels. This in turn can cause significant cut-off to the collateral blood supply and pose a particular risk of immediate ischemia to the ganglionic structures supplied by the perforator vessels. In large M1 segment MCA occlusions a non-engaged suction catheter within the terminal ICA/proximal M1 MCA segment could cause flow reversal within the collaterals supplied by the ACA and enhance the prospect of worsening the ischemia ${ }^{8}$.

We hypothesize that differences in clinical outcomes of aspiration thrombectomy in published case series may be due to variations in technique between operators. If suction is discontinued and the device repositioned, little or no blood will be removed from the collaterals/cerebral circulation. However, if suction is continued in the same place with continued good flow in the suction catheter when the clot is not engaged in the catheter tip there is no aspiration force on the clot and collaterals may be put at risk. The amount of blood aspirated is limited by the volume of the syringes $(30-50$ $\mathrm{ml}$ ) with manual suction. However, if a pump is used, larger volumes of blood can be removed. This may be particularly significant for aspiration in the M1 and basilar 
artery, as in these areas collaterals are in close proximity to the suction tip and could be put at risk by a reversal in flow.

Our conclusions and the resulting hypothesis are based on mathematical modelling and in vitro experiments in a glass model of the cerebral circulation. Our findings need to be confirmed by in vivo experiments and by observation of clinical scenarios. The 4F catheter used in these experiments is at the lower range of sizes used for intracranial suction thrombectomy (ID 0.89-1.63) in clinical practice 202324 but appropriate for the size of the experimental vessel. The diameter of the catheter has no bearing on the conclusions of this work, as the results are qualitative rather than quantitative. However, as flow rates increase exponentially with increasing catheter diameter, the observed effects on flow would be expected to be greater with larger bore devices, unless the devices are so large as to restrict flow in the vessel.

In vitro simulations and experiments provide explanatory data, which can help to optimize interventional technique. Our results suggest that for aspiration thrombectomy to be effective, the catheter must be in direct contact with the thrombus. Until there is clear evidence that flow reversal by suction is not a problem in vivo, the suction catheter should not be kept in one location for a prolonged period of time during aspiration when there is constant flow, as there may be a risk of reducing pressure and flow in the collateral vessels. We would also recommend close monitoring of pump suction to limit the amount of blood removal to an absolute minimum during one suction episode. This may not be relevant in large vessel occlusions without collaterals nearby. Use of wider bore aspiration catheters, which 
occlude the vessel lumen proximally, could also reduce the risk of affecting collateral flow. This has recently been described as the ADAPT FAST technique ${ }^{17}$.

1

2

3

4

5

6

7

8

9

10

11

12

13

14

15

16

17

18

19

20

21

22

23

24

25

26

27

28

29

30

31

32

33

34

35

36

37

38

39

40

41

42

43

44

45

46

47

48

49

50

51

52

53

54

55

56

57

58

59

60 


\section{References}

1. Rha JH, Saver JL. The Impact of Recanalization on Ischemic Stroke Outcome: A Meta-Analysis. Stroke 2007;38(3):967-73.

2. Saqqur M, Uchino K, Demchuk AM, et al. Site of Arterial Occlusion Identified by Transcranial Doppler Predicts the Response to Intravenous Thrombolysis for Stroke. Stroke 2007;38(3):948-54.

3. Berkhemer OA, Fransen PS, Beumer D, et al. A randomized trial of intraarterial treatment for acute ischemic stroke. N Engl J Med 2015;372(1):11-20.

4. Campbell BC, Mitchell PJ, Kleinig TJ, et al. Endovascular therapy for ischemic stroke with perfusion-imaging selection. N Engl J Med 2015;372(11):1009-18.

5. Goyal M, Demchuk AM, Menon BK, et al. Randomized assessment of rapid endovascular treatment of ischemic stroke. N Engl J Med 2015;372(11):101930.

6. Jovin TG, Chamorro A, Cobo E, et al. Thrombectomy within 8 Hours after Symptom Onset in Ischemic Stroke. N Engl J Med 2015.

7. Saver JL, Goyal M, Bonafe A, et al. Solitaire ${ }^{\mathrm{TM}}$ with the Intention for Thrombectomy as Primary Endovascular Treatment for Acute Ischemic Stroke (SWIFT PRIME) trial: protocol for a randomized, controlled, multicenter study comparing the Solitaire revascularization device with IV tPA with IV IPA alone in acute ischemic stroke. International Journal of Stroke 2015;10(3):439-48.

8. Lally F, Grunwald IQ, Sanyal R, et al. Mechanical thrombectomy in acute ischaemic stroke: a review of the literature, clinical effectiveness and future use. CNS Neurol Disord Drug Targets 2013;12(2):170-90. 
9. Becktepe JS, You SJ, Berkefeld J, et al. Clinical Outcome after Mechanical Recanalization as Mono- or Adjunctive Therapy in Acute Stroke: Importance of Time to Recanalization. Cerebrovascular Diseases 2011;32(3):211-18.

10. Bose A, Henkes H, Alfke K, et al. The Penumbra System: A Mechanical Device for the Treatment of Acute Stroke due to Thromboembolism. American Journal of Neuroradiology 2008;29(7):1409-13.

11. Taschner CA, Treier MF, Schumacher MF, et al. Mechanical thrombectomy with the Penumbra recanalization device in acute ischemic stroke. J Neuroradiol 2011(0150-9861 (Print)).

12. The Penumbra Pivotal Stroke Trial I. The Penumbra Pivotal Stroke Trial. Stroke 2009;40(8):2761-68.

13. Grunwald IQ, Walter S, Papanagiotou P, et al. Revascularization in acute ischaemic stroke using the penumbra system: the first single center experience. European Journal of Neurology 2009;16(11):1210-16.

14. Jankowitz B, Aghaebrahim A, Zirra A, et al. Manual Aspiration Thrombectomy. Stroke 2012.

15. Kang DH, Hwang YH, Kim YS, et al. Direct Thrombus Retrieval Using the Reperfusion Catheter of the Penumbra System: Forced-Suction Thrombectomy in Acute Ischemic Stroke. American Journal of Neuroradiology 2011;32(2):283-87.

16. Kulcsar Z, Bonvin C, Pereira VM, et al. Penumbra System: A Novel Mechanical Thrombectomy Device for Large-Vessel Occlusions in Acute Stroke. American Journal of Neuroradiology 2010;31(4):628-33. 
17. Turk AS, Frei D, Fiorella D, et al. ADAPT FAST study: a direct aspiration first pass technique for acute stroke thrombectomy. J Neurointerv Surg 2014;6(4):260-4.

18. Tsai JP, Albers GW. Reperfusion Versus Recanalization: The Winner Is.... Stroke 2015;46:2.

19. Tennuci C, Pearce G, Wong J, et al. Comparison of the Effectiveness of Three Methods of Recanalization in a Model of the Middle Cerebral Artery: Thrombus Aspiration via a 4F Catheter, Thrombus Aspiration via the GP Thromboaspiration Device, and Mechanical Thrombectomy Using the Solitaire Thrombectomy Device. Stroke Research And Treatment 2011;2011:18642424.

20. Kreusch AS, Psychogios M-N, Knauth M. Techniques and Results-Penumbra Aspiration Catheter. Techniques in Vascular and Interventional Radiology 2012;15(1):53-59.

21. Penumbra Inc. Alameda C, 5701, rev A. Instructions for use. Penumbra System®.

22. Symon L, Ishikawa S, Meyer JS. Cerebral arterial pressure changes and development of leptomeningeal collateral circulation. Neurology 1963;13:23750.

23. Jankowitz BT, Aleu A, Lin R, et al. Endovascular treatment of basilar artery occlusion by manual aspiration thrombectomy. Journal of Neurolnterventional Surgery 2010;2(2):110-14.

24. Lutsep HL, Clark WM, Nesbit GM, et al. Intraarterial Suction Thrombectomy in Acute Stroke. American Journal of Neuroradiology 2002;23(5):783-86. 
1

2

3

4

5

6

7

8

9

10

11

12

13

14

15

16

17

18

19

20

21

22

23

24

25

26

27

28

29

30

31

32

33

34

35

36

37

38

39

40

41

42

43

44

45

46

47

48

49

50

51

52

53

54

55

56

57

58

59

60

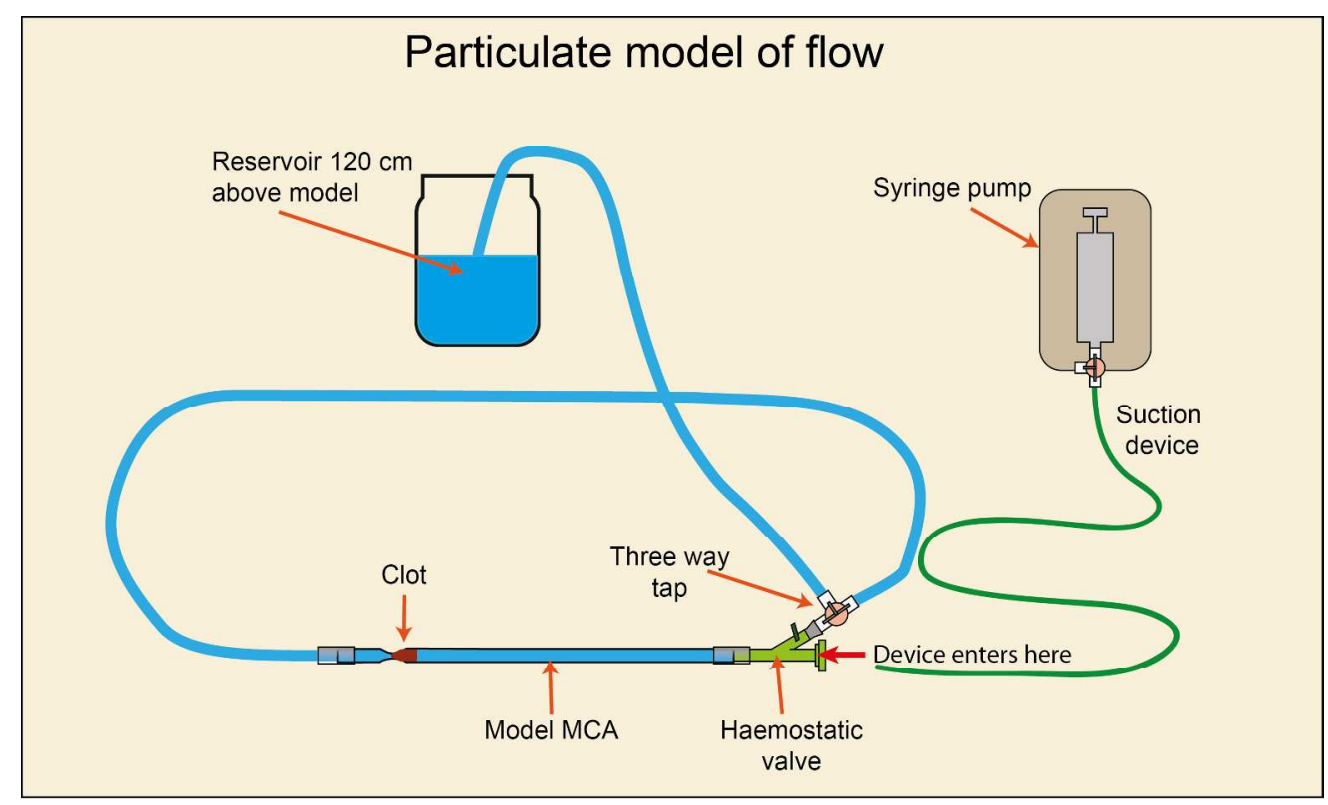

Figure 1: Diagrammatic view of the particulate system incorporating a glass model of the MCA with a pinch point for the thrombus. The system was primed at a mean arterial pressure of $90 \mathrm{~mm} \mathrm{Hg}$ by elevating the fluid reservoir $122 \mathrm{~cm}$ above the model.

$250 \times 150 \mathrm{~mm}(300 \times 300 \mathrm{DPI})$ 


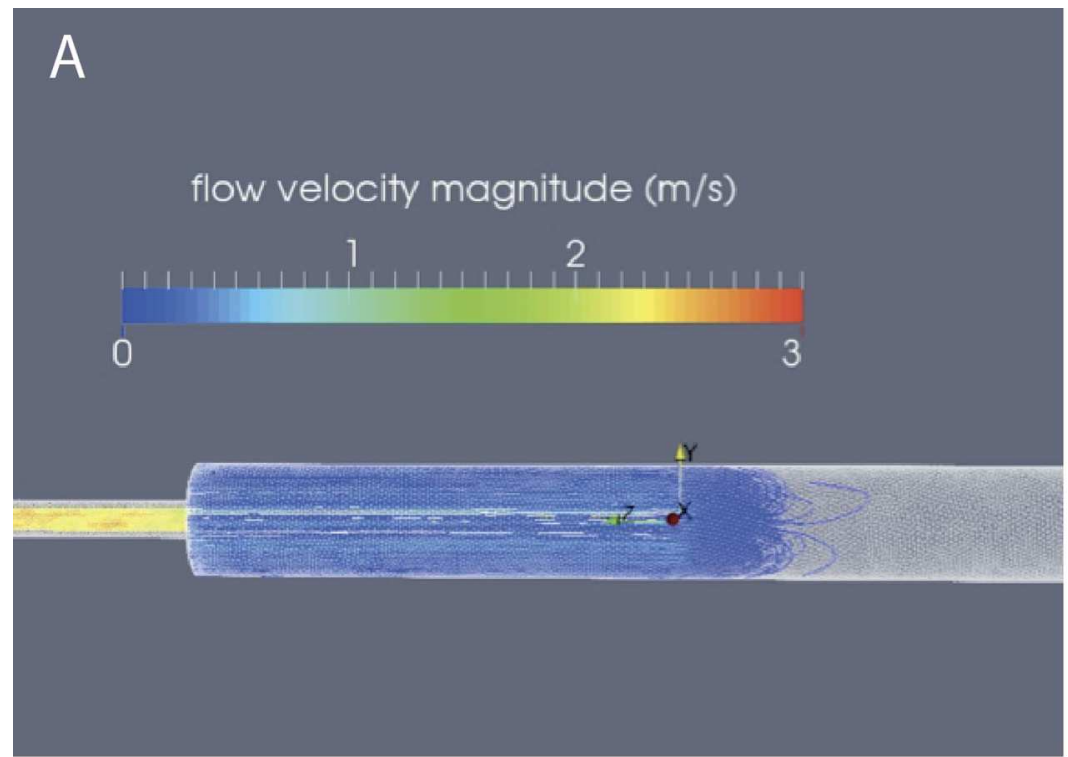

\section{B}

\section{Flow velocity magnitude $(\mathrm{m} / \mathrm{s})$}

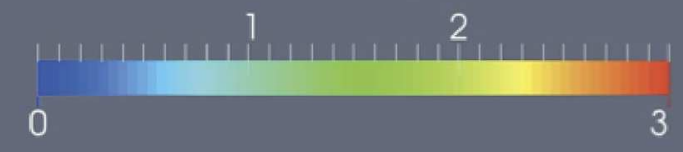

Figure 2: (a) Output from mathematical model showing torsional movement downstream of catheter tip but no torsional movement upstream of the catheter tip. (b) Shows an unoccluded model with clear flow from the same seed points upstream of the catheter tip as well as flow from the negative $z$ direction. $107 \times 157 \mathrm{~mm}(300 \times 300$ DPI $)$ 

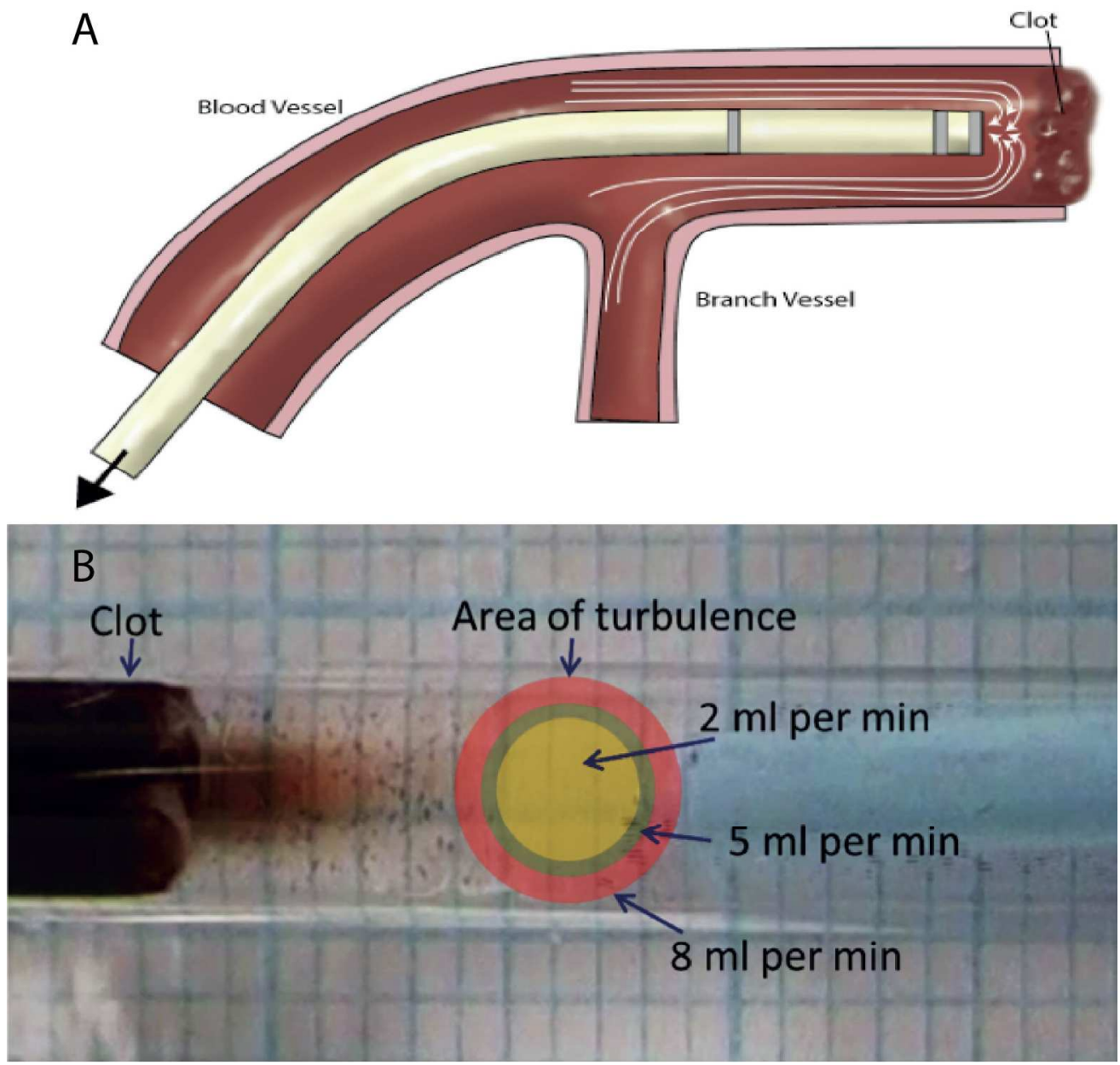

Figure 3: (a) Graphical representation of flow as shown in the mathematical model.

(b) Area of turbulence in an occluded model seen at all flow rates and separation distances. The turbulence increased with increasing flow rates and did not engage the clot at any distance from the catheter. $202 \times 194 \mathrm{~mm}(300 \times 300$ DPI) 
Figure 4: (a) Flow only occurred from behind the tip of the device when suction was applied to devices in an occluded model, (b) In an unoccluded model, The main flow came from in front of the catheter tip but there was still a clear although less dominant flow present from behind the catheter tip. $154 \times 207 \mathrm{~mm}(300 \times 300$ DPI $)$ 
Manuscript ID neurintsurg-2015-011909.R1 entitled "In vitro experiments of cerebral blood flow during aspiration thrombectomy" - Associate editor's comments and author's responses

\begin{tabular}{|c|c|}
\hline Associate editor's comments (a) & Responses (b) \\
\hline $\begin{array}{l}\text { 1.1 Please consider modifying the title of your } \\
\text { manuscript to make it more explicit to your } \\
\text { hypothesis and/or results. It may help other } \\
\text { researchers who are researching the technical } \\
\text { aspects of aspiration thrombectomy to locate } \\
\text { your paper. }\end{array}$ & $\begin{array}{l}\text { We have changed the title to: } \\
\text { 'In vitro experiments of cerebral blood flow } \\
\text { during aspiration thrombectomy: Potential } \\
\text { effects on cerebral perfusion pressure and } \\
\text { collateral flow.' }\end{array}$ \\
\hline $\begin{array}{l}\text { 1.2 I think one area still being sorted out for } \\
\text { mechanical thrombectomy, regardless of } \\
\text { whether a separator, stent-triever or the } \\
\text { ADAPT technique is being used, is at what } \\
\text { point to turn on the aspiration, or begin } \\
\text { manual aspiration. } \\
\text { I appreciate your elaboration in the discussion, } \\
\text { but it might be worthwhile for your } \\
\text { neurointerventionalists to comment on the } \\
\text { clinical relevance of your findings in the } \\
\text { context of the current conventional approaches } \\
\text { to aspiration thrombectomy. } \\
\text { Your study does give in vitro evidence that } \\
\text { this should be performed more thoughtfully } \\
\text { and carefully. }\end{array}$ & $\begin{array}{l}\text { We believe that we have fully described the } \\
\text { clinical relevance of the findings of our study } \\
\text { which is that it is only safe to apply suction } \\
\text { when the device is in contact with the thrombus. } \\
\text { To further clarify this point we have added a } \\
\text { sentence (see below) to the final paragraph of } \\
\text { the discussion making clear the potential } \\
\text { consequences of suction when the device is not } \\
\text { in contact with the thrombus: } \\
\text { '......as there may be a risk of reducing pressure } \\
\text { and flow in the collateral vessels.' }\end{array}$ \\
\hline
\end{tabular}




\section{In vitro experiments of cerebral blood flow during aspiration}

\section{thrombectomy: Potential effects on cerebral perfusion pressure}

and collateral flow.

Keywords: Stroke, recanalization, thrombectomy, aspiration, blood flow

Word count: 2140 


\begin{abstract}
Background: Mechanical thrombectomy with stenttriever devices is associated with significantly better outcomes than thrombolysis alone in the treatment of acute ischaemic stroke. Thrombus aspiration achieves high patency rates, but clinical outcomes are variable. The aim of this study was to examine the effect of different suction conditions on perfusate flow during aspiration thrombectomy.
\end{abstract}

Materials: A computational fluid dynamics model of an aspiration device within a patent and occluded blood vessel was used to simulate flow characteristics using fluid flow solver software (OpenFOAM, OpenCFD Ltd). A physical particulate-flow model of a patent vessel and a vessel occluded by thrombus was then used to visualize flow direction and measure flow rates with the aspiration catheter placed 1$10 \mathrm{~mm}$ proximal of the thrombus, and recorded on video.

Results: The mathematical model predicted that in a patent vessel perfusate is drawn from upstream of the catheter tip, while in an occluded system perfusate is drawn from the vessel proximal to the device tip, with no traction on the occlusion distal of the tip. The in-vitro experiments confirmed the predictions of this model. In the occluded vessel aspiration had no effect on the thrombus unless the tip of the catheter was in direct contact with the thrombus.

Conclusion: These experiments suggest that aspiration is only effective if the catheter tip is in direct contact with the thrombus. If the catheter tip is not in contact with the thrombus aspirate is drawn from the vessels proximal of the occlusion. This could affect collateral flow in vivo. 


\section{Introduction}

Early restoration of blood flow to the ischaemic brain is pivotal to good recovery and improved outcomes in ischaemic stroke patients ${ }^{1}$. Recanalization rates with intravenous thrombolysis are poor in patients with occlusion of a large intracerebral artery $(30 \%$ in basilar artery occlusions, $30 \%$ in proximal middle cerebral artery occlusions and $6 \%$ in terminal internal carotid artery occlusions) ${ }^{2}$. Endovascular treatment $(E V T)$ is associated with considerably higher recanalization rates than intravenous thrombolysis ${ }^{1}$. The most commonly used methods for EVT are mechanical thrombectomy using a stenttriever device and thrombus aspiration. Recently presented randomized controlled trials have shown significantly better outcomes for mechanical thrombectomy compared to control when stentrievers were used $^{3-7}$. Aspiration devices achieve consistently high recanalization rates (68-100\%), but clinical outcomes with aspiration are variable ${ }^{8}$ with very poor recovery rates in four studies ${ }^{9-12}$ and good outcomes in others ${ }^{13-17}$. Recanalization alone may not ensure good outcomes, if reperfusion is not achieved ${ }^{18}$.

In vitro experiments can be a useful tool for the study of specific attributes of device performance. Experiments in an in vitro model have previously shown that suction thrombectomy is considerably faster than thrombectomy using a stenttirever device but associated with a higher incidence of thrombus fragmentation ${ }^{19}$.

The aim of this study was to examine perfusate flow patterns during aspiration thrombectomy in an in-vitro system to investigate the effects of different suction conditions (flow rate, suction pressure, and distance from the thrombus) on flow in a model of occluded and unoccluded cerebral arteries. 


\section{Methods}

A mathematical model of blood flow in a blocked cerebral blood vessel was

developed. A physical flow model of suction thrombectomy in an occluded and unoccluded vessel was then set up to visualize flow patterns and to assess the effects of different aspiration conditions in vitro.

\section{The mathematical model}

A computational fluid dynamics (CFD) model was used to simulate the fluid flow characteristics of a typical catheter used for our experiments. A model of a cylinder within a cylinder was chosen to represent a plain catheter within an artery. The dimensions of the catheter cylinder were based on diameters of the catheters used (see below); approximately $1.4 \mathrm{~mm}$ OD and $0.89 \mathrm{~mm}$ ID. The artery cylinder was approximately $3 \mathrm{~mm}$ in diameter and the distal surface positioned $10 \mathrm{~mm}$ from the distal tip of the catheter cylinder. This model assumed laminar flow based on calculated Reynolds numbers that were in the order of thousands. Geometries of the fluid-flow system were created, meshed and run using the fluid-flow solver OpenFOAM (@ OpenCFD Ltd.).

\section{The physical flow model}

The model was set up as in Figure 1 incorporating a glass model of the MCA with an internal diameter of $2.4 \mathrm{~mm}$ and a downstream pinch point diameter of $2 \mathrm{~mm}$ where the thrombus was placed. A haemostatic valve proximal to the glass model was used to introduce catheters into the system. The system was adapted from a model described previously ${ }^{19}$ and primed at a mean arterial pressure of $90 \mathrm{~mm} \mathrm{Hg}$ by 
elevating the fluid reservoir $122 \mathrm{~cm}$ above the model. Two devices were used to create suction within the system; a syringe pump (Fusion 200 Touch syringe pump, KR Analyitical Ltd, UK) was used for generating low flow rates and a vacuum pump (Welch Vacuum Technology 2546B-01) for higher flow rates.

The system was primed with perfusate containing haematoxylin particles to allow visualization of flow patterns. This was prepared as follows: $1 \mathrm{ml}$ of haematoxylin solution (Gill no.3 - GHS316-Sigma Aldrich) was added to $800 \mathrm{ml}$ of water and left for 1 hour to produce clumps. The solution was stirred and allowed to settle for 30 minutes repeatedly to obtain a suspension containing smaller particles. This produced a particulate that behaved like a colloid over the time periods of the experiments without significant settling and allowed even small movements of flow to be observed.

\section{Thrombus production}

Fresh sheep's blood was obtained from a local abattoir in a closed container. The blood was allowed to clot naturally. After clotting the blood was cut into small pieces and snap-frozen in liquid nitrogen before being placed in a freezer at $-20^{\circ} \mathrm{C}$. Thrombi were taken from the freezer in small quantities and allowed to thaw gradually in physiological saline solution at $4^{\circ} \mathrm{C}$. On thawing the thrombus was cut down with a scalpel until it weighed between 12 - $14 \mathrm{~g}$ after drying on a paper towel. This weight had been arrived at experientially and provided a clot of a size that engaged well within the model system. Once primed, the clot was introduced into the system and pushed to the stricture point using brief flow from the pump. The model was then 
flushed with perfusate to remove solubilised blood from around the clot to allow accurate visualisation.

\section{Flow observation in a blocked system}

A catheter was introduced into the system via the haemostatic valve, with the tip at a distance of $1,2,2.5$, and $10 \mathrm{~mm}$ from the clot. Air was removed from the system and the particulate allowed to equilibrate. At an appropriate point the pump was switched on and the flow in the area of the catheter tip and clot was observed over a minimum of 30 seconds using a video camera (Samsung, 42X INTELLI-ZOOM, 24fps). Flow rates used ranged from 5 to $40 \mathrm{kPa}$ using the vacuum pump and 2,5 and $8 \mathrm{ml} / \mathrm{min}$ using the syringe pump. The flow rates were calculated by analysis of video data from the closed system. Fifteen particles were randomly selected and time taken for each particle to travel $2 \mathrm{~cm}$ was timed using a stopwatch.

\section{Catheters}

Two catheters were used, a general purpose Fr4 MC 0.035" (0.89mm) (Kimal, Uxbridge, UK) and a NeuroSlider*17 (0.42mm) microcatheter (Acandis, Pforzheim, Germany). The size of the catheter was chosen to match the size of the target vessel (2.4 $\mathrm{mm}$ ) using recommendations for catheter size for currently used catheters in vivo $^{2021}$.

\section{Results}

\section{Mathematical model of suction thrombectomy}

Results of the modelling showed that in an unobstructed system (cerebral artery not occluded by a thrombus) flow is drawn from upstream of the tip of a device when 
suction is applied. In an occluded system (cerebral artery obstructed by a thrombus) the mathematical model showed that flow came from behind the tip of the device when suction was applied, and that there was no traction effect on flow distal of the tip. The scaled-up stream tracer plots of an occluded model in Figure 2a show torsional movement downstream of catheter tip but no visible torsional movement from seed points $3 \mathrm{~mm}$ upstream of the catheter tip. The only $\mathrm{x}$ and $\mathrm{y}$-direction flow pattern is seen as the fluid flows past the outside of the catheter in the negative zdirection, bending around the distal end of the catheter tip toward the inside of the device. The tracer plots in figure $2 \mathrm{~b}$ which comes from the unoccluded mathematical model shows clear flow from the same $3 \mathrm{~mm}$ seed points upstream of the catheter tip as well as flow from the negative $z$ direction.

\section{Physical model}

At all flow rates and separation distances, fluid was clearly drawn from behind the catheter as predicted by the mathematical model (Figure 2) and not from in front when a clot was present, which is illustrated in Figure 3a. As the flow became steady an area of turbulence was observed in front of the catheter tip and this area of turbulence increased with increasing flow rates (Figure 3b). The turbulence had no effect on clot removal at any distance from the catheter, the catheter needed to be touching the clot before suction had any effect. At further separation distances, no clot movement or engagement with the suction catheter was observed in any of the experiments.

When suction was applied to the devices in an occluded model, flow only occurred from behind the tip of the device, Figure 4a. When suction was applied to the devices in the absence of a thrombus flow from the front of the device was restored 
but there was still a clear but less dominant flow present from behind the catheter tip, Figure $4 b$.

\section{Discussion}

Mathematical modelling of blood flow in an occluded vessel during suction through a catheter showed an area of turbulence between the clot and the catheter tip with blood drawn from the vessel segment proximal to the catheter tip and no effect of the suction on the clot or on the vessel segment distal to the clot. These findings from our mathematical model are confirmed by in-vitro experiments performed in a flow model.

The key finding of this in-vitro research is that catheter-based suction only engaged the thrombus if the tip of the catheter was in direct contact with the thrombus. When the thrombus was not engaged in the tip of the suction catheter, suction removed only perfusate, and produced no traction forces on the thrombus. Perfusate removed by suction came from behind, e.g. the vessel section proximal to the catheter tip. This has potentially significant implications for clinical practice.

\footnotetext{
After occlusion of a large artery, the ensuing drop in perfusion pressure distally generates a pressure gradient between neighbouring arterial fields, resulting in changes in flow direction and rate; collateral flow changes occur almost immediately, within 1-4 seconds ${ }^{22}$. In our experimental model we observed that non-engagement of the suction catheter with the thrombus results in removal of the perfusate proximal
} 
of the catheter tip. In a clinical scenario this could lead to flow reversal in the proximal/collateral vessels, leading to a decrease in the perfusion pressure within the ischemic penumbra. This in turn would produce a vicious cycle of further reduction of blood flow in other collateral vessels, thereby incorporating a significant proportion of the salvageable penumbra into the ischemic core. Such flow reductions could be particularly relevant during aspiration in small calibre M2 segment of the middle cerebral artery (MCA) or in the basilar artery. Placement of the aspiration catheter within the distal M1/proximal M2 could cause flow reversal within the other viable MCA branches including the lateral and medial lenticulostriate perforator vessels. This in turn can cause significant cut-off to the collateral blood supply and pose a particular risk of immediate ischemia to the ganglionic structures supplied by the perforator vessels. In large M1 segment MCA occlusions a non-engaged suction catheter within the terminal ICA/proximal M1 MCA segment could cause flow reversal within the collaterals supplied by the ACA and enhance the prospect of worsening the ischemia ${ }^{8}$.

We hypothesize that differences in clinical outcomes of aspiration thrombectomy in published case series may be due to variations in technique between operators. If suction is discontinued and the device repositioned, little or no blood will be removed from the collaterals/cerebral circulation. However, if suction is continued in the same place with continued good flow in the suction catheter when the clot is not engaged in the catheter tip there is no aspiration force on the clot and collaterals may be put at risk. The amount of blood aspirated is limited by the volume of the syringes $(30-50$ $\mathrm{ml}$ ) with manual suction. However, if a pump is used, larger volumes of blood can be removed. This may be particularly significant for aspiration in the M1 and basilar 
artery, as in these areas collaterals are in close proximity to the suction tip and could be put at risk by a reversal in flow.

Our conclusions and the resulting hypothesis are based on mathematical modelling and in vitro experiments in a glass model of the cerebral circulation. Our findings need to be confirmed by in vivo experiments and by observation of clinical scenarios. The 4F catheter used in these experiments is at the lower range of sizes used for intracranial suction thrombectomy (ID 0.89-1.63) in clinical practice 202324 but appropriate for the size of the experimental vessel. The diameter of the catheter has no bearing on the conclusions of this work, as the results are qualitative rather than quantitative. However, as flow rates increase exponentially with increasing catheter diameter, the observed effects on flow would be expected to be greater with larger bore devices, unless the devices are so large as to restrict flow in the vessel.

In vitro simulations and experiments provide explanatory data, which can help to optimize interventional technique. Our results suggest that for aspiration thrombectomy to be effective, the catheter must be in direct contact with the thrombus. Until there is clear evidence that flow reversal by suction is not a problem in vivo, the suction catheter should not be kept in one location for a prolonged period of time during aspiration when there is constant flow, as there may be a risk of reducing pressure and flow in the collateral vessels. We would also recommend close monitoring of pump suction to limit the amount of blood removal to an absolute minimum during one suction episode. This may not be relevant in large vessel occlusions without collaterals nearby. Use of wider bore aspiration catheters, which 
occlude the vessel lumen proximally, could also reduce the risk of affecting collateral flow. This has recently been described as the ADAPT FAST technique ${ }^{17}$. 


\section{References}

1. Rha JH, Saver JL. The Impact of Recanalization on Ischemic Stroke Outcome: A Meta-Analysis. Stroke 2007;38(3):967-73.

2. Saqqur M, Uchino K, Demchuk AM, et al. Site of Arterial Occlusion Identified by Transcranial Doppler Predicts the Response to Intravenous Thrombolysis for Stroke. Stroke 2007;38(3):948-54.

3. Berkhemer OA, Fransen PS, Beumer D, et al. A randomized trial of intraarterial treatment for acute ischemic stroke. N Engl J Med 2015;372(1):11-20.

4. Campbell BC, Mitchell PJ, Kleinig TJ, et al. Endovascular therapy for ischemic stroke with perfusion-imaging selection. N Engl J Med 2015;372(11):1009-18.

5. Goyal M, Demchuk AM, Menon BK, et al. Randomized assessment of rapid endovascular treatment of ischemic stroke. N Engl J Med 2015;372(11):101930.

6. Jovin TG, Chamorro A, Cobo E, et al. Thrombectomy within 8 Hours after Symptom Onset in Ischemic Stroke. N Engl J Med 2015.

7. Saver JL, Goyal M, Bonafe A, et al. Solitaire ${ }^{\mathrm{TM}}$ with the Intention for Thrombectomy as Primary Endovascular Treatment for Acute Ischemic Stroke (SWIFT PRIME) trial: protocol for a randomized, controlled, multicenter study comparing the Solitaire revascularization device with IV IPA with IV IPA alone in acute ischemic stroke. International Journal of Stroke 2015;10(3):439-48.

8. Lally F, Grunwald IQ, Sanyal R, et al. Mechanical thrombectomy in acute ischaemic stroke: a review of the literature, clinical effectiveness and future use. CNS Neurol Disord Drug Targets 2013;12(2):170-90. 
9. Becktepe JS, You SJ, Berkefeld J, et al. Clinical Outcome after Mechanical Recanalization as Mono- or Adjunctive Therapy in Acute Stroke: Importance of Time to Recanalization. Cerebrovascular Diseases 2011;32(3):211-18.

10. Bose A, Henkes H, Alfke K, et al. The Penumbra System: A Mechanical Device for the Treatment of Acute Stroke due to Thromboembolism. American Journal of Neuroradiology 2008;29(7):1409-13.

11. Taschner CA, Treier MF, Schumacher MF, et al. Mechanical thrombectomy with the Penumbra recanalization device in acute ischemic stroke. J Neuroradiol 2011(0150-9861 (Print)).

12. The Penumbra Pivotal Stroke Trial I. The Penumbra Pivotal Stroke Trial. Stroke 2009;40(8):2761-68.

13. Grunwald IQ, Walter S, Papanagiotou P, et al. Revascularization in acute ischaemic stroke using the penumbra system: the first single center experience. European Journal of Neurology 2009;16(11):1210-16.

14. Jankowitz B, Aghaebrahim A, Zirra A, et al. Manual Aspiration Thrombectomy. Stroke 2012.

15. Kang DH, Hwang YH, Kim YS, et al. Direct Thrombus Retrieval Using the Reperfusion Catheter of the Penumbra System: Forced-Suction Thrombectomy in Acute Ischemic Stroke. American Journal of Neuroradiology 2011;32(2):283-87.

16. Kulcsar Z, Bonvin C, Pereira VM, et al. Penumbra System: A Novel Mechanical Thrombectomy Device for Large-Vessel Occlusions in Acute Stroke. American Journal of Neuroradiology 2010;31(4):628-33. 
17. Turk AS, Frei D, Fiorella D, et al. ADAPT FAST study: a direct aspiration first pass technique for acute stroke thrombectomy. J Neurointerv Surg $2014 ; 6(4): 260-4$.

18. Tsai JP, Albers GW. Reperfusion Versus Recanalization: The Winner Is.... Stroke 2015;46:2.

19. Tennuci C, Pearce G, Wong J, et al. Comparison of the Effectiveness of Three Methods of Recanalization in a Model of the Middle Cerebral Artery: Thrombus Aspiration via a 4F Catheter, Thrombus Aspiration via the GP Thromboaspiration Device, and Mechanical Thrombectomy Using the Solitaire Thrombectomy Device. Stroke Research And Treatment 2011;2011:18642424.

20. Kreusch AS, Psychogios M-N, Knauth M. Techniques and Results-Penumbra Aspiration Catheter. Techniques in Vascular and Interventional Radiology 2012;15(1):53-59.

21. Penumbra Inc. Alameda C, 5701, rev A. Instructions for use. Penumbra System®.

22. Symon L, Ishikawa S, Meyer JS. Cerebral arterial pressure changes and development of leptomeningeal collateral circulation. Neurology 1963;13:23750.

23. Jankowitz BT, Aleu A, Lin R, et al. Endovascular treatment of basilar artery occlusion by manual aspiration thrombectomy. Journal of Neurolnterventional Surgery 2010;2(2):110-14.

24. Lutsep HL, Clark WM, Nesbit GM, et al. Intraarterial Suction Thrombectomy in Acute Stroke. American Journal of Neuroradiology 2002;23(5):783-86. 